\title{
AN ANALYSIS OF THE VARIATIONAL INEQUALITIES OF SEEPAGE FLOW BY FINITE-ELEMENT METHODS*
}

\author{
BY \\ NOBORU KIKUCHI \\ University of Texas, Austin
}

1. Introduction. In this decade the theory of variational inequalities has been developed by a number of prominent mathematicians. At present we can find in the literature not only the elements of a mathematical theory, but also several applications to mechanics and physics; see for example, Duvaut and Lions [1]. Historically, this theory started in an investigation of a Signorini problem in elasticity by Fichera [2]. The beginning of the development of a systematic mathematical framework for this theory is generally linked with the work of Lions and Stampacchia [3]. The relationship between the free boundary problems and the theory of variational inequalities has been explicitly investigated by Lions [4].

In this paper, we study this relationship in some detail using a typical free boundary problem, namely the analysis of seepage flow, and we investigate the approximation theory of variational inequalities. We also show how error estimates of approximations of variational inequalities by finite-element methods can be obtained, and we compare the results obtained by variational inequalities with the results of Taylor's method which was developed to find the free surface in seepage flow problems. The present work may be regarded as an expository article designed to demonstrate in simple terms the basic qualitative properties of the theory of variational inequalities and to show an application of the theory to a typical example problem involving the free boundary.

The use of variational inequalities in connection with seepage flow problems was deeply studied by Baiocchi $[5,6,7]$ and others. In this paper, we use the simplest case of Baiocchi's theory. Numerical resolution of this problem was performed by Comincioli $[8,9]$ and others. The error estimates of approximation of a class of variational inequalities were investigated by Falk [10]. Here, we study a modification of Falk's theory. This theory can be applied to all kinds of variational inequalities provided we approximate closed convex sets with subsets. We elaborate on this requirement later in the paper.

In Sec. 2, we explain the mathematical theory of variational inequalities. First the equivalence between variational inequalities and free boundary problems is explained using a simple example. Second, we give an existence theorem which suggests how we calculate the variational inequality numerically. Third, in order to interpret physically the meaning of the solution, we introduce a regularity theorem for variational inequalities and illustrate it by means of a simple example. Then we establish error estimates of finiteelement approxımations of variational inequalities which are confirmed by numerical experiments.

* Received April 8, 1976; revised version received July 14, 1976. The support of this work by a grant from N.S.F. is gratefully acknowledged. 
In Sec. 3, we explain the method of application of variational inequalities to mechanics. Specifically, we describe how transformations can be made which are necessary for formulating a given problem in terms of variaitonal inequalities. The considerations also are helpful in solving other problems, which include free boundaries and various discontinuity (jump) conditions, using the idea of variational inequalities. Finally, we compare the numerical results of the variational inequality method associated with seepage flow problems with the numerical results of Taylor's method [11]. From this comparison, we are able to identify certain advantages and disadvantages of the formulation.

\section{Mathematical theory of variational inequalities.}

2.1 Existence and regularity theorem. Let $H$ be a reflexive Banach space and $K$ be a closed convex subset of $H$. Let $A$ be an operator from $H$ into the dual space $H^{\prime}$ of $H$. The following problem is said to be variational inequality (or unilateral problem): find $u \in K$ such that, for $f \in H^{\prime}$,

$$
\langle A u-f, v-u\rangle \geq 0, \quad \forall v \in K .
$$

In the present study, it suffices to consider only those cases in which $H$ is a real Hilbert space.

If $A$ is a potential operator of a functional $F$ on $H$, then (2.1) is equivalent to the constrained minimization problem: find $u \in K$ such that

$$
F(u)-2\langle f, u\rangle \leq F(v)-2\langle f, v\rangle, \quad \forall v \in K .
$$

An important aspect of variational inequalities is that if the admissible set $K$ is represented by some inequality-type constrained conditions, then variational inequalities may be equivalent to the free boundary problems. In order to confirm this aspect, an example could help. Let $H^{m}(G)$ be the $m$ th-order Sobolev space on $G$.

Example 1. Consider the variational inequality: $u \in K$,

$$
\int_{0}^{1}\left\{u,_{x}(v-u)_{,_{x}}+(v-u)\right\} d x \geq 0 \text { for } \forall v \in K
$$

where $K=\left\{v \mid v \in H^{1}(0,1), v(0)=0.25, v(1)=0, v \geq 0\right.$ a.e. in $\left.(0,1)\right\}$. As shown later, (2.3) has a unique solution $u$ belonging to $H^{2}(0,1)$. Then by integration by parts (2.3) can be written as

$$
\int_{0}^{1}\left(-u_{x_{x}}+1\right)(v-u) d x \geq 0, \quad \forall v \in K .
$$

Let $\delta(0)$ be a mollifer of the Dirac $\delta$-function with $\delta(0)=1$ at $x=0$. Clearly, $0.25 \delta(0)$ and $2 u-0.25 \delta(0)$ belong to $K$. Then

$$
\int_{0}^{1}\left(-u,_{x r}+1\right)( \pm u) d x \geq 0 .
$$

This means that

$$
\left(-u,_{x x}+1\right) u=0 \quad \text { a.e. in }(0,1) .
$$

By (2.4), 


$$
\int_{0}^{1}\left(-u,_{x x}+1\right) v d x \geq \int_{0}^{1}\left(-u,_{x x}+1\right) u d x=0 .
$$

Since $v \geq 0$ a.e. in $(0,1),-u,_{x x}+1 \geq 0$ a.e. in $(0,1)$. Therefore, $(2.3)$ is equivalent to

$$
\left.\begin{array}{r}
u \geq 0 \\
\left(-u_{x_{x}}+1\right) u=0 \\
-u_{x_{x x}}+1 \geq 0 \\
(0)=0.25, u(1)=0
\end{array}\right\} \quad \text { a.e. in }(0,1)
$$

Thus, there are two parts in $(0,1)$ such that $\Omega_{+}=\{x \mid u(x)>0\}$ and $\Omega_{0}=\{x \mid u(x)=0\}$. Since $u$ is the regular solution, (2.5) is equivalent to

$$
\begin{array}{r}
-u_{x_{x x}}+1=0 \quad \text { in } \quad(0, p) \\
u=0 \quad \text { in } \quad(p, 1)
\end{array}
$$

where $0<p<1$. In this case $p=1 / \sqrt{ } 2$. It is also worthwhile to note that if another boundary condition is imposed, the "free" boundary $p$ may not occur. For example, if $K^{\prime}=\left\{v \mid v \in H^{1}(0,1), v(0)=1, v(1)=0, v \geq 0\right.$ a.e. in $\left.(0,1)\right\}$, then the solution of $(2.3)$ is

$$
u(x)=0.5(x-1.5)^{2}-1 / 8>0 \text { in }(0,1) .
$$

Now we note the existence theorem of variational inequalities following Lions and Stampacchia [3]. Let $P_{K}$ be the Riesz's projection map from $H$ onto $K,(\cdot, \cdot)$ an inner product on $H$.

Lemma 1. (a) $u=P_{K} f$ is equivalent to (b) $(u-f, v-u) \geq 0$ for $\forall v \in K$.

Theorem 1. (c) $u \in K$ is a solution of

$$
u=P_{K}(u-t(A u-f)), \quad t>0
$$

is equivalent to (d) $u \in K$ is a solution of

$$
(A u-f, v-u) \geq 0 \text { for } \forall v \in K .
$$

Furthermore, if $A$ is linear, coercive, and continuous on $H$, i.e. there are positive constants $M$ and $m$ such that

$$
\begin{aligned}
& (A u, v) \leq M\|u\|\|v\| \\
& (A u, u) \geq m\|u\|^{2}
\end{aligned}
$$

for every $u, v$ in $H$, then the operator $T: T u=P_{K}(u-t(A u-f))$ is a contraction map on $H$ for constants $0<t<2 m / M^{2}$. This means that there exists a unique solution $u$ in $H$ of $(2.8)$.

Proof. The equivalence (c) and (d) is obvious from Lemma 1. Since $\left\|P_{K} u-P_{K} v\right\| \leq$ $\|u-v\|$,

$$
\begin{aligned}
\left\|{ }^{\prime} u_{1}-T u_{2}\right\| & \leq\left(u_{1}-u_{2}-t\left(A u_{1}-A u_{2}\right), u_{1}-u_{2}-t\left(A u_{1}-A u_{2}\right)\right) \\
& \leq\left(1-2 m t+M^{2} t^{2}\right)\left\|u_{1}-u_{2}\right\|^{2} .
\end{aligned}
$$

Thus, if $0<t<2 m / M^{2}, 0<1-2 m t+M^{2} t^{2}<1$. This means that $T$ is a contraction. 
Remark 1. Here we assumed that $A$ is linear, but $A$ is not necessarily linear. In fact, if there exist constants $m$ and $M$ such that

$$
\begin{aligned}
(A u-A v, u-v) & \geq m\|u-v\|^{2} \\
(A u-A v, u) & \leq M\|u-v\|\|w\|
\end{aligned}
$$

for $u, v$ and $w$ in $H$, then the same conclusion in Theorem 1 is valid.

Remark 2. The equivalence between (c) and (d) suggests that we can solve Eq. (2.7) instead of the inequality (2.8). When $P_{K}=1$, the scheme of (2.7) is called the method of steepest descents. That is, the solution $u$ can be obtained by the iteration

$$
u^{(n+1)}=P_{K}\left(u^{(n)}-t\left(A u^{(n)}-f\right)\right) \text {. }
$$

Note that if $K=\{v \mid v \in H, M v \geq g$ a.e. in $\Omega\}$ where $g$ is some given function and $M$ is operator, then the projection map is, roughly speaking, considered as

$$
P_{K}(v)=\operatorname{Max}\left\{v, M^{-1} g\right\} \text {. }
$$

In variational inequalities, the regularity of solutions is much complicated since the constraint conditions are imposed. About this subject there is a remarkable work by Brezis [12]. We state here his theorem:

Theorem 2 (Brezis). Let $C$ be a closed convex subset of $L_{2}(\Omega)$ and $L$ a closed convex subset of a Hilbert space $V . P_{C}$ denotes the projection map of $L_{2}(\Omega)$ onto $C$. Suppose that (i) $P_{C}(L) \subset L$, (ii) there exists a positive constant $C_{1}$ such that, for every $v \in L$, $\left(A v, v-P_{C} v\right) \geq-C_{1}\left\|v-P_{C} v\right\|_{L_{2}(\Omega)}$, (iii) there is a unique solution $u \in H^{2}(\Omega) \cap L$ of,

$$
(A u-f, v-u) \geq 0, \quad \forall v \in L
$$

for $f \in L_{2}(\Omega)$. Further,

$$
\|u\|_{H^{2}(\Omega)} \leq C_{3}\left(\|f\|_{L_{2}(\Omega)}+C_{2}\right) .
$$

Then, for every $f \in L_{2}(\Omega)$, there exists a unique solution $u \in H^{2}(\Omega) \cap C \cap L$ of

$$
(A u-f, v-u) \geq 0, \quad \forall v \in C \cap L
$$

and

$$
\|u\|_{H^{2}(\Omega)} \leq C_{3}\left(2\|f\|_{L_{2}(\Omega)}+C_{1}+C_{2}\right) .
$$

Example 2. Let's consider the problem (2.3), i.e.

$$
u \in K ; \quad \int_{0}^{1}\left\{u,_{x}(v-u)_{{ }_{x}}+(v-u)\right\} d x \geq 0 \text { for } \forall v \in K
$$

where $K=\left\{v \mid v \in H^{1}(0,1), v(0)=0.25, v(1)=0, v(x) \geq 0\right.$ in $\left.(0,1)\right\}$. Since there is $f \in H^{2}(0,1)$ such that $f(0)=0.25$ and $f(1)=0,(2.3)$ can be changed as follows: find $w \in K^{\prime}$ such that

$$
\int_{0}^{1}\left\{w,_{x}(v-w),_{x}+\left(1-f,_{x x}\right)(v-w)\right\} d x \geq 0, \quad \forall v \in K^{\prime}
$$

where $K^{\prime}=\left\{v \mid v \in H_{0}{ }^{1}(0,1), v(x) \geq-f(x)\right.$ a.e. in $\left.(0,1)\right\}$. Now we apply Theorem 2 . Let $C=K, L=V=H_{0}{ }^{1}(0,1)$. Then, clearly $P_{K}(L) \subset L$. Since $P_{K} v=\sup \{v,-f\}$ and $v-P_{K} v=-\sup \{0,-v-f\}$, 


$$
\begin{aligned}
\int_{0}^{1} v,_{x}\left(v-P_{K} v\right),_{x} d x & =-\int_{0}^{1} v_{\cdot x} \sup \{0,-v-f\},_{x} d x \\
& =\int_{0}^{1}(-f-v)_{,_{x}} \sup \{0,-v-f\},_{x} d x+\int_{0}^{1} f,_{x} \sup \{0,-v-f\},_{x} d x \\
& \geq-\int_{0}^{1} f_{,_{x}}\left(v-P_{K} v\right)_{,_{x}} d x=\int_{1}^{1} f,_{x_{x}}\left(v-P_{K} v\right) d x \\
& \geq-\left\|f,_{,_{x}}\right\|_{L_{2}(\Omega)}\left\|v-P_{K} v\right\|_{L_{2}(\Omega)} .
\end{aligned}
$$

Moreover, by the regularity of boundary-value problems, there exists $v \in H_{0}{ }^{1}(0,1) \cap$ $H^{2}(0,1)$ of

$$
\int_{0}^{1}\left\{w,_{x}(v-w)_{x}+\left(1-f,_{x}\right)(v-w)\right\} d x \geq 0, \quad \forall v \in H_{0}{ }^{\prime}(0,1) ;
$$

that is,

$$
\int_{0}^{1}\left\{w,_{x} v_{,_{x}}+\left(1-f,_{x_{x}}\right)\right\} d x=0, \quad \forall v \in H_{0}{ }^{\prime}(0,1)
$$

and

$$
\|w\|_{H^{2}(0,1)} \leq C\left(\left\|1-f_{x_{x}}\right\|_{L_{2}(\Omega)}\right) .
$$

Therefore, (2.12) has a unique solution $w \in K^{\prime} \cap H_{0}{ }^{1}(0,1) \cap H^{2}(0,1)$ such that

$$
\|w\|_{H^{2}(\Omega)} \leq 3 C\left\|f_{x_{x}}\right\|_{L,(\Omega)} .
$$

2.2 Approximation theory. Let $H_{h}$ be a finte-dimensional subsapce of $H$ with basis $\left\{\phi_{i}\right\}_{i=1}^{N}$ constructed by finite-element methods. Let us define the approximated closed convex subset $K_{h}$ by $K_{h}=K \cap H_{h}$. Suppose that $K_{h} \rightarrow K$ and $H_{h} \rightarrow H$ as $h \rightarrow 0$, where $h$ is the mesh parameter of finite elements.

Lemma 2. Let $u$ and $U$ be solutions of

$$
(A u-f, v-u) \geq 0 \text { for } \forall v \in K
$$

and

$$
(A U-f, V-U) \geq 0 \text { for } \forall V \in K_{h}
$$

respectively. Then

$$
(A u-f, V-u) \geq(A u-A U, V-U)
$$

for every $V \in K_{h}$.

Proof. $(A u-f, V-U)=(A U-f, V-U)+(A u-A U, V-U)$. By (2.15),

$$
(A u-f, V-U) \geq(A u-A U, V-U) .
$$

Since $U \in K$,

$$
(A u-f, U-u) \geq 0
$$

by (2.14). Add (2.16) and (2.17):

$$
(A u-f, V,-u) \geq(A u-A U, V-U)
$$

Suppose that $L$ is a dense Hilbert space in $H$. 
Theorem 3: Let $u$ and $U$ be solutions of (2.14) and (2.15), respectively. If $A$ is linear, coercive and continuous, then there exist $m$ and $M$ such that

$$
\|u-U\|_{H^{2}} \leq \frac{M^{2}}{m^{2}}\|u-V\|_{H}{ }^{2}+\frac{2}{m}\|f-A u\|_{L}\|u-V\|_{L}
$$

for every $V \in K_{h}$.

Proof. Since $A$ is coercive, there is a positive constant $m$ such that

$$
\begin{aligned}
m\|u-U\|_{u}{ }^{2} & \leq(A u-A U, u-U) \\
& =(A u-A U, u-V)+(A u-A U, V-U) .
\end{aligned}
$$

By Lemma 2 ,

$$
m\|u-U\|_{n}{ }^{2} \leq(A u-A U, u-V)+(f-A u, u-V) .
$$

By continuity of $A$, there is a positive constant $M$ such that

$$
\begin{aligned}
m\|u-U\|^{2} & \leq M\|u-U\|_{n}\|u-V\|_{H}+(f-A u, u-V) \\
& \leq \frac{m}{2}\|u-U\|_{u}{ }^{2}+\frac{M^{2}}{2 m}\|u-V\|_{H}{ }^{2}+\|f-A u\|_{L}\|u-V\|_{L}
\end{aligned}
$$

The approximation error above is bounded only by norms of $u-V, V \in K_{h}$. Then, in order to get the error estimates, it is not necessary to make any special consideration except interpolation properties of functions. Thus, we specify interpolation properties to accomplish the error estimates.

For this purpose we introduce the $S_{h}{ }^{k, m}$-family as an approximation space of $H$. The ${S_{h}}^{k, m}$-family has the following properties:

(a) $S_{h}{ }^{k, m} \subset H^{m}(\Omega)$.

(b) $\mathcal{P}_{k}(\Omega) \subset S_{h}{ }^{k, m}$, where $\mathcal{P}_{k}(\Omega)$ is the space of polynomials of degree $\leq k$ on $\Omega$.

(c) For every $u \in H^{r}(\Omega)$ and $0 \leq s \leq \min \{m, r\}$, there exist $\pi u \in S_{h}{ }^{k, m}$ and positive constant $C$, independent of $u$ and $h$, such that

$$
\|u-\pi u\|_{H^{*}(\Omega)} \leq C h^{\sigma}\|u\|_{\left.H^{r}(i)\right)}, \quad \sigma=\min \{k+1-s, r-s\} .
$$

Suppose that the approximated space $H_{h}$ is a member of the $S_{h}{ }^{k, m}$-families. Then, $H_{h} \rightarrow H$ as $h \rightarrow 0$ and $H_{h}$ has the interpolation property. However, $K_{h}$ is a problem in variational inequalities. That is, construction of $K_{h}$ as $K \cap H_{h}$ becomes a serious problem. We see this by considering an example.

Example 3. Let us consider the approximation of the problem (2.3). Suppose that $H_{h}$ is a member of $S_{h}{ }^{1,1}$. Then $K_{h}$ can be constructed as

$$
K_{h}=\left\{V \mid V=\sum_{i=1}^{N} V^{i} \phi_{i}, V^{1}=0.25, V^{N}=0, V^{i} \geq 0\right\} .
$$

Clearly, $K_{h}=K \cap H_{h}$. However, if $H_{h}$ is a member of ${S_{h}}^{2,1}$, then the above construction does not satisfy $K_{h} \subset K$. That is, if $V^{i}=a>0, V^{i+1}=0$, and $V^{i+2}=0$, then in the interval $(i+1, i+2), V \leq 0$. Here, we consider only Lagrange interpolation-type finiteelement methods. The underlying explanation is only for linear finite-element approximations. As shown in Example 2, (2.3) has a unique solution $u \in H^{2}(0,1) \cap K$. For the same reason, the approximation problem of (2.3) has a unique solution $U$ in $K_{h}$. Using Theorem 3 , we can get the following approximation error: 


$$
\|u-U\|_{H^{1}(0,1)}{ }^{2} \leq \frac{M^{2}}{m^{2}}\|u-V\|_{H^{1}(0,1)}{ }^{2}+\frac{2}{m}\|f-A u\|_{L_{2}(0,1)}\|u-V\|_{L_{2}(0,1)} .
$$

By the interpolation property (2.19) with $k=1$ and $r=2$,

$$
\begin{aligned}
& \|u-\pi u\|_{H^{1}(0,1)} \leq C h\|u\|_{H^{2}(0,1)}, \\
& \|u-\pi u\|_{L_{2}(0,1)} \leq C h^{2} \mid i u \|_{I^{2}(1,1)} .
\end{aligned}
$$

Therefore,

$$
\|u-U\|_{H^{\prime}(0,1)}^{2} \leq C\left(\frac{M^{2}}{m^{2}}\|u\|_{H^{2}(0,1)}^{2}+\frac{2}{m}\|f-A u\|_{L_{2}(1,1)}\|u\|_{H^{2}(0,1)}\right) h^{2} .
$$

That is, $\|u-U\|_{H^{1}(0,1)}{ }^{2}=O(h)$. Thus, we get the same rate of convergence as variational boundary value problems.

The numerical experiment by the author shows the above result exactly; cf. Fig. 1.

3. Seepage flow problems with finite-element methods. As an example of variational inequalities, we will consider seepage flow through a porous medium. This kind of treatment has been deeply studied by Baiocchi $[5,6,7]$ and others. Here it suffices to work the simplest case of Baiocchi's studies for our purpose so that we may unify the

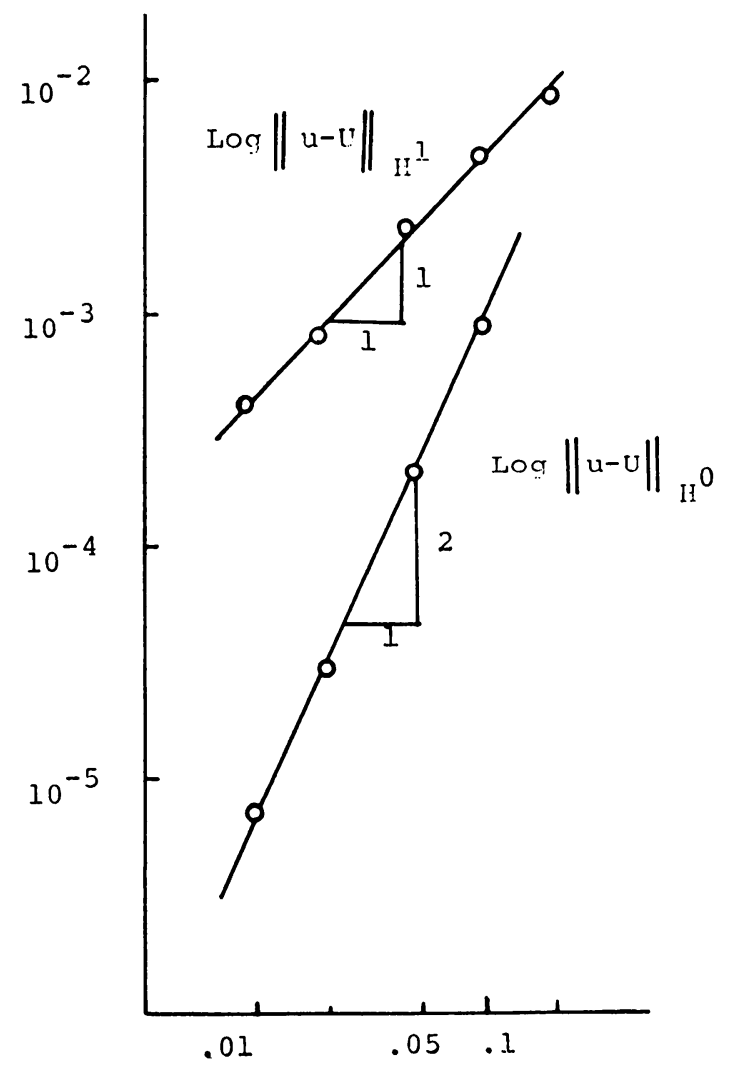

FIg. 1. Numerical results of error estimates for one-dimensional problem. 
method of applications of variational inequalities. Then we discuss why the special transformation introduced by Baiocchi is necessary to formulate the variational inequality. After the formulation we compare the numerical results of this problem with the usual iterative method to get the free surface introduced by Taylor [11].

Assume that the porous medium is isotropic and homogeneous, and the flow is stationary, irrotational and incompressible. Then the seepage flow is formulated by the continuity equation and Darcy's law as

$$
\partial^{2} u / \partial x^{2}+\partial^{2} u / \partial y^{2}=0 \text { in } \Omega
$$

where $u$ is the piezometric head. As in Fig. 2, let $\phi(x)$ be the free surface which is unknown a priori. In the usual sense, the boundary conditions are

$$
\begin{aligned}
u=y_{1} \text { on } \overparen{A E}, \quad u=y_{2} \text { on } \overparen{C B}, \quad \frac{\partial u}{\partial n}=0 \text { on } \overparen{A B}, \\
u=\phi(x) \text { on } \overparen{E D C}, \quad \frac{\partial u}{\partial u}=0 \text { on } \overparen{E D .}
\end{aligned}
$$

Theorem 4. Let the pair $\{u, \phi\}$ be the solution of the problem (3.1)-(3.3); then for $0<x<a$ the integral

$$
q(x)=\int_{0}^{\phi(x)} \frac{\partial u}{\partial x}(x, t) d t
$$

exists and is constant.

Proof. Since $u$ satisfies (3.1),

$$
0=\int_{0}^{a} \int_{0}^{\phi(x)} \nabla u \cdot \nabla v d \mathbf{x}
$$

for every $v \in C_{0}{ }^{\infty}\left(\Omega_{a}\right), \Omega_{a}=(0, a) \otimes(0, \phi(x))$.

Since $v(x, y) \in C_{0}{ }^{\infty}\left(\Omega_{a}\right)$ can be decomposed by

$$
v(x, y)=s(x) \cdot 1(y)
$$

where $S(x) \in C_{0}{ }^{\infty}(0, a)$, and $1(y)$ is a "constant" distribution on $(0, \phi(x))$. Then

$$
0=-\int_{0}^{a} \frac{d}{d x}\left[\int_{0}^{\phi(x)} \frac{\partial u}{\partial x} d y\right] s(x) d x .
$$

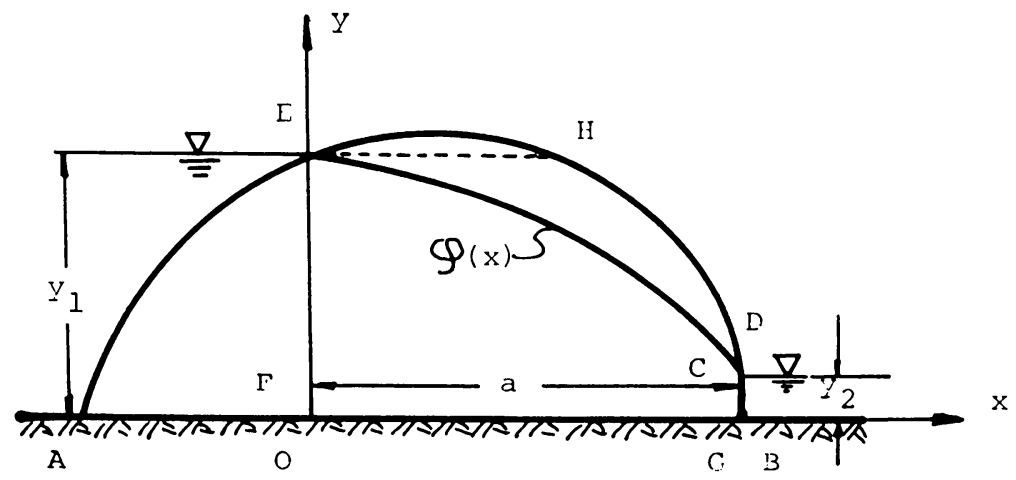

FIG. 2. Domain for Theorem 4. 
Thus, in the sense of distribution,

$$
0=\frac{d}{d x} \int_{0}^{\phi(x)} \frac{\partial u}{\partial x} d y \text {, i.e. } \int_{0}^{\phi(\lrcorner)} \frac{\partial u}{\partial x} d y=\text { const. }
$$

Note that the above theorem is only valid when the impermeable foundation is horizontal or at least straight.

Now, we first consider the simplest case: the cross-section of the porous medium is a rectangle, as shown in Fig. 3. Using the special transformation introduced by Baiocchi

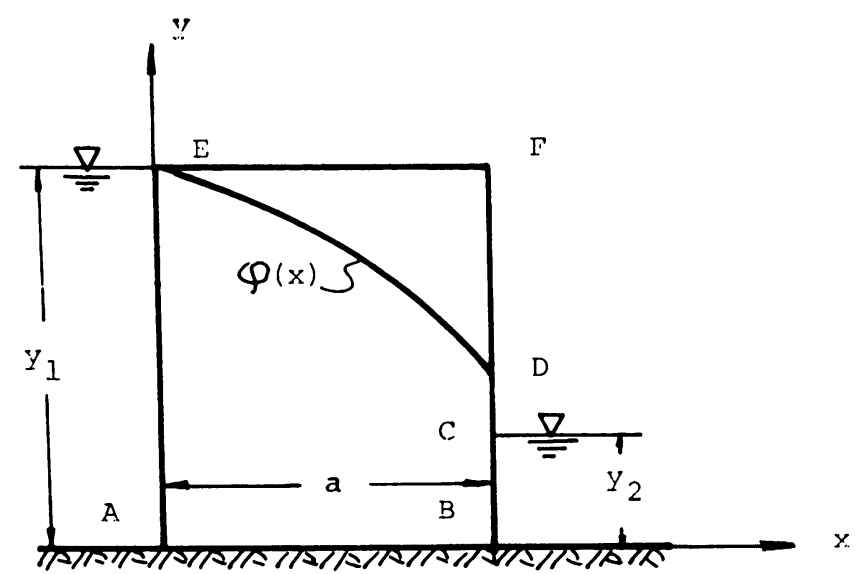

FIG. 3. Domain for Lemma 4 and Theorem 5.

$$
\begin{aligned}
& w(x, y)=\int_{\nu}^{\nu_{1}}(u(x, t)-t) d t \quad \text { for } \quad(x, y) \in \bar{\Omega} \\
& =0 \quad \text { for }(x, y) \in \bar{D} / \bar{\Omega},
\end{aligned}
$$

where $\bar{D}=$ the domain of the rectangle $A B F E$, we can get the following Lemma 3:

Lemma 3. Let the pair $\{u, \phi\}$ be the solution of the problem (3.1)-(3.3). Then

(1) $\quad w(x, y) \geq 0$ a.e. in $D$ and $u(x, y) \in H^{1}(D)$;

(2) $\quad w(x, y)=g(x, y)$ on $\partial D$, where $g=\frac{1}{2}\left(y_{1}-y\right)^{2}$ on $\overparen{A E}$,

$$
\begin{array}{r}
g=\frac{1}{2}\left(y_{2}-y\right)^{2} \text { on } \overparen{B C,} \quad g=\frac{1}{2} y_{1}{ }^{2}-\frac{1}{2 a}\left(y_{1}{ }^{2}-y_{2}{ }^{2}\right) x \text { on } \overparen{A B}, \\
\text { and } g=0 \text { on } \overparen{E F D C} . \\
\text { (3) } \quad \int_{D} \nabla u \cdot \nabla \psi d x d y+\int_{\Omega} \psi d x d y=0 \text { for } \forall \psi \in H_{0}{ }^{1}(D)
\end{array}
$$

Proof. (1) Since $u(x, y) \geq y$ in $\bar{\Omega}, w(x, y) \geq 0$ and $u(x, y) \in H^{1}(\Omega), w(x, y)$ is defined by (3.5). Furthermore $w(x, y)$ is continuous and the first derivatives of $w(x, y)$ are piecewise continuous; then $w(x, y) \in H^{1}(D)$.

(2) Using Theorem 4, we can get, by simple calculation,

(3) Since $\psi(x, y) \in H_{0}{ }^{1}(D), \eta(x, y)=\int_{0}{ }^{y} \psi(x, t) d t \in H_{0}{ }^{1}(D)$, then 


$$
\begin{gathered}
\int_{D} \nabla u \cdot \nabla \psi d x d y=\int_{\Omega} \nabla u \cdot \nabla \psi d x d y=-\int_{\Omega} \nabla\left(\frac{\partial w}{\partial y}\right) \cdot \nabla \eta d x d y \\
\int_{\Omega} \nabla u \cdot \nabla \eta d x d y-\int_{\Omega} \frac{\partial \eta}{\partial y} d x d y=-\int_{\Omega} \psi d x d y .
\end{gathered}
$$

Note that condition (1) is the essential property to formulate by the variational inequality. First, $u(x, y) \geq 0$ means that in the fixed domain $w(x, y)$ is greater than zero or equal to zero. More precisely, if $w(x, y)>0$ then $(x, y) \in \Omega$; if $w(x, y)=0$ then $(x, y) \in \bar{D} / \bar{\Omega}$. Second, $u(x, y)$ belongs to $H^{1}(D)$, that is the value $w(x, y)$ and its first derivatives are piecewise continuous in $D$. This means that we can naturally extend $u(x, y)$ defined only in an unknown $\Omega$ to $w(x, y)$ defined in the fixed domain $D$ without any problems which we may face at the time of the formulation in the variational form. If $w(x, y)$ or its first derivatives have discontinuity on the unknown surface $E D$, some "unknown" terms will appear in the variational form (3.8). Thus, in order to extend the unknown domain to the fixed known domain, the variable should be extended as maintaining the continuity of the variable itself and its first derivatives in the second-order differential equations. This means that if we can find the transformation which preserves the "continuity", then we can extend the problem by this transformation. For example, the transformation

$$
\begin{aligned}
z(x, y) & =\int_{y}^{\prime \prime}(u(x, t)-t) d t & \text { in } \bar{\Omega} \\
& =z(x, \phi(x)) & \text { in } \bar{l} / \bar{\Omega}
\end{aligned}
$$

also satisfies the "continuity" condition explained above, i.e. if $u(x, y) \in H^{1}(\Omega)$, then $z(x, y) \in H^{1}(D)$. For details of this transformation, see Baiocchi [7].

Therefore, from Lemma 3, we can formulate the seepage flow by variational inequalities as follows:

Theorem 5. If the pair $\{u, \phi\}$ is the solution of (3.1)-(3.3), then the following variational inequality is established:

$$
w \in K: \int_{D} \nabla w \cdot \nabla(v-u) d x d y+\int_{D}(v-w) d x d y \geq 0
$$

for all $v \in K$, where $K=\left\{v\left|v \in H^{1}(\Omega), v\right|_{\partial D}=g, v \geq 0\right.$ a.e. in $\left.D\right\}$.

Furthermore, as explained in Examples 1 and 2, the solution $w$ of (3.10) gives the solution $u$ of (3.1)-(3.3), that is, the problem (3.1)-(3.3) is exactly equivalent to the variational inequality (3.10). Thus, after obtaining the solution $w$ of (3.10), we find

$$
\begin{gathered}
\Omega=\{(x, y) \in D: w(x, y)>0\} \\
\phi(x)=\operatorname{Min}_{y_{2}<t<y_{1}}\{t: w(x, t)=0,0 \leq x \leq a\} \\
(x, y) \text { at } D: y_{D}=\lim _{\rightarrow \rightarrow 0} \phi(a-\epsilon) \\
u=y-\frac{\partial w}{\partial y} \text { in } \Omega .
\end{gathered}
$$

Then, applying the technique cited in Remark 2, we can calculate the variational 
inequality of (3.10) by iteration. More precisely, after approximation by the linear finite element method, the iteration is defined by:

Algorithm 1. (1) Select an arbitrary set of elements $W_{(0)}$ which belongs to $K_{h}$ (possibly $W_{(n)}=0$ ).

$$
W_{(t+1)}{ }^{k}=\max \left\{0, W_{(\ell)^{k}}{ }^{k}-\rho\left(\sum_{j=1}^{k-1} K_{k j} W_{(\ell+1)}{ }^{i}+\sum_{j=k}^{N} K_{k j} W_{(\ell)^{i}}{ }^{i}-F_{k}\right\}\right.
$$

where

$$
K_{k i}=\int_{0} \nabla \phi_{k} \nabla \phi_{i} d x d y, \quad F_{k}=-\int_{D} \phi_{k} d x d y
$$

and $\left\{\phi_{i}\right\}_{i=1}^{N}$ is basis of $S_{h}$.

(3) The recurrence formula (3.12) is applied successfully until covnergence is obtained.

In this case, we can get the approximation error $e=w-W$ as

$$
\|e\|_{H^{1}(D)}=O(h) \text { and }\|e\|_{L_{2}(D)}=O\left(h^{2}\right),
$$

as shown in Example 3.

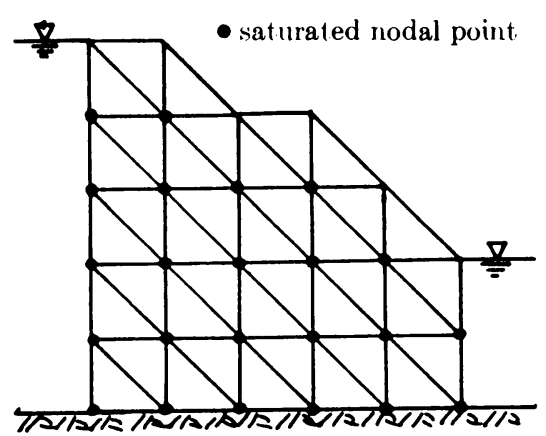

4a. Free surface by variational inequalities (element $2 \times 5 \times 5$ ).

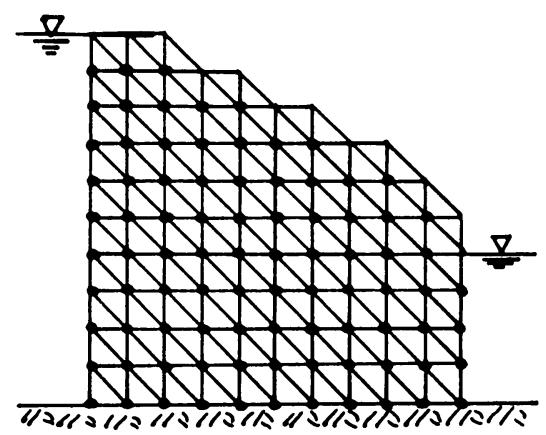

4b. Free surface by variational inequalities (element $2 \times 10 \times 10$ ).

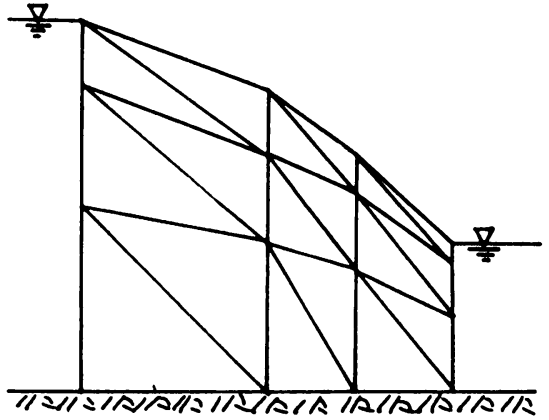

4c. Free surface by Taylor's method (element $2 \times 3 \times 3$ ).

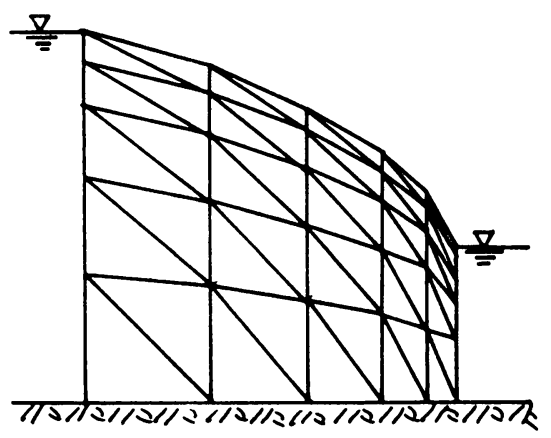

4d. Free surface by Taylor's method (element $2 \times 5 \times 5$ ).

Fig. 4. 
Now we describe the numerical test of the variational inequality. For comparison with this method we use Taylor's method [11] to determine the free surface using iteration of domains satisfying the condition $\partial u / \partial n=0$ for the free surface $\overparen{E D C}$. This convergence can be determined by the condition $u(x, y)=y$ on $\overparen{E D C}$. Then we can conclude the following:

(1) The method of variational inequalities gives a very rough free surface if the number of meshes is small (Fig. 4). In order to get a suitable smooth surface (Fig. 5), it needs more than ten times the number of Taylor's method. However, in Taylor's method, the system of linear equations has to be solved several times to get convergence. In the method of variational inequalities, it is not necessary to solve the system of linear equations more than one time.

(2) The method of variational inequalities can determine the seepage point $D$
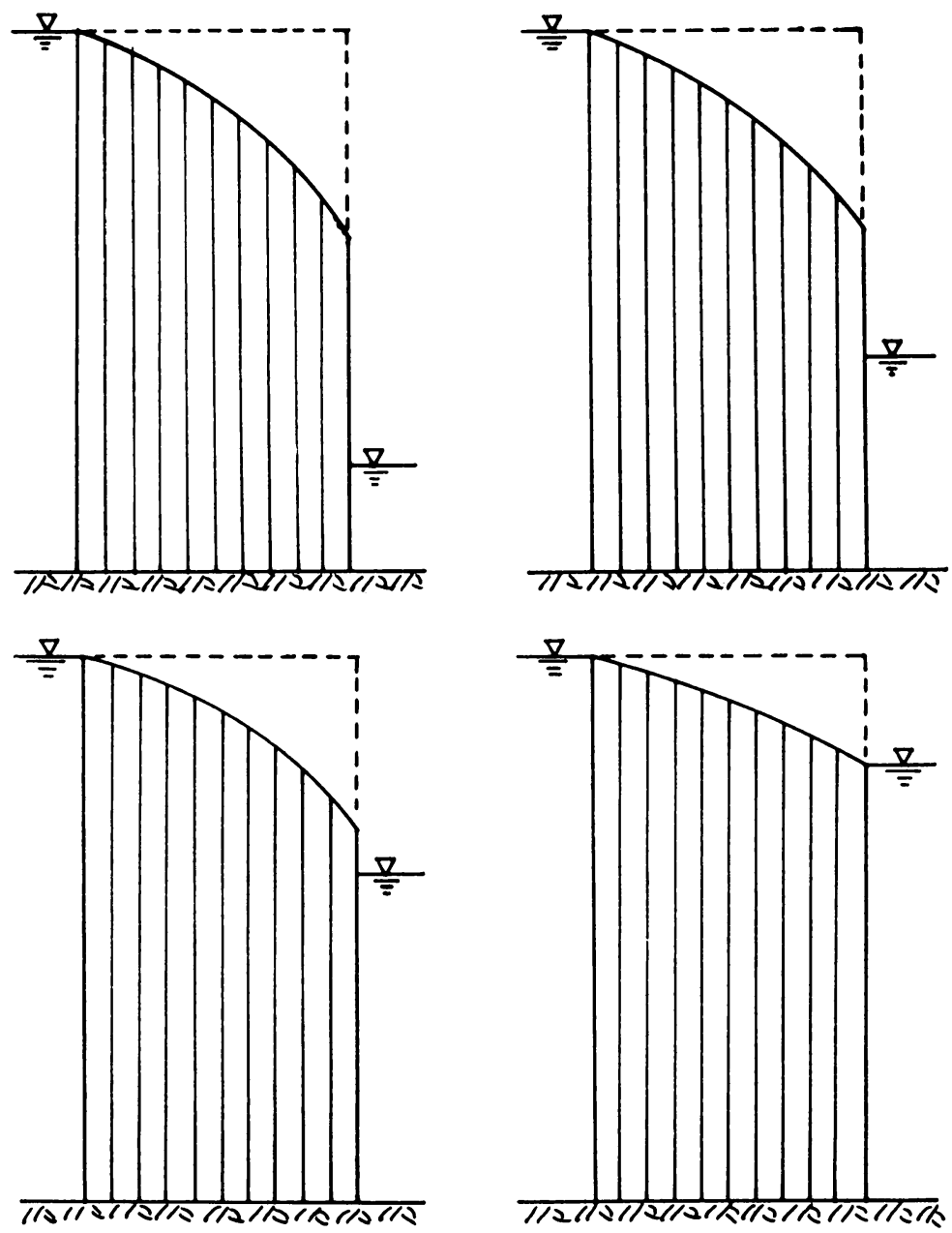

FIg. 5. Free surface by variational inequalities (element $2 \times 20 \times 40$ ). 
(cf. Fig. 3) without ony special considerations. However, Taylor's method needs some special considerations (see [11]).

(3) Taylor's method has no guarantee of convergence, but the method of variational inequalities does.

According to the numerical experiment by the author, the method of variational inequalities is not so powerful from a numerical viewpoint. From the theroetical viewpoint, however, this method suggests many things to us. For example, we can treat ambiguous conditions precisely using this theory. This means that almost all free boundary problems can be formulated by the variational inequalities within the rigorous theory. The possibilities of applications for mechanics are discussed by Duvaut and Lions [1].
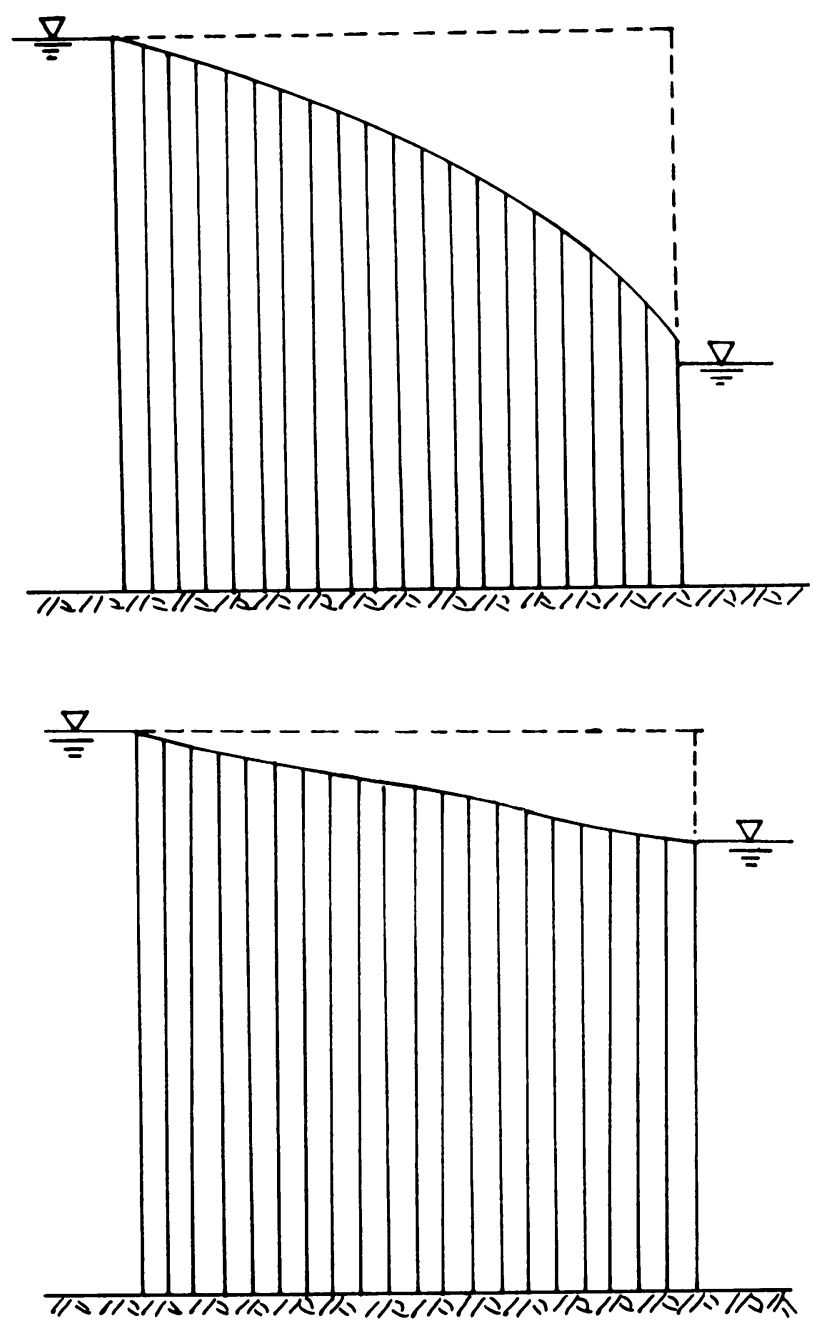

FIg. 6a. Free surface by variational inequality (3200 finite elements). 

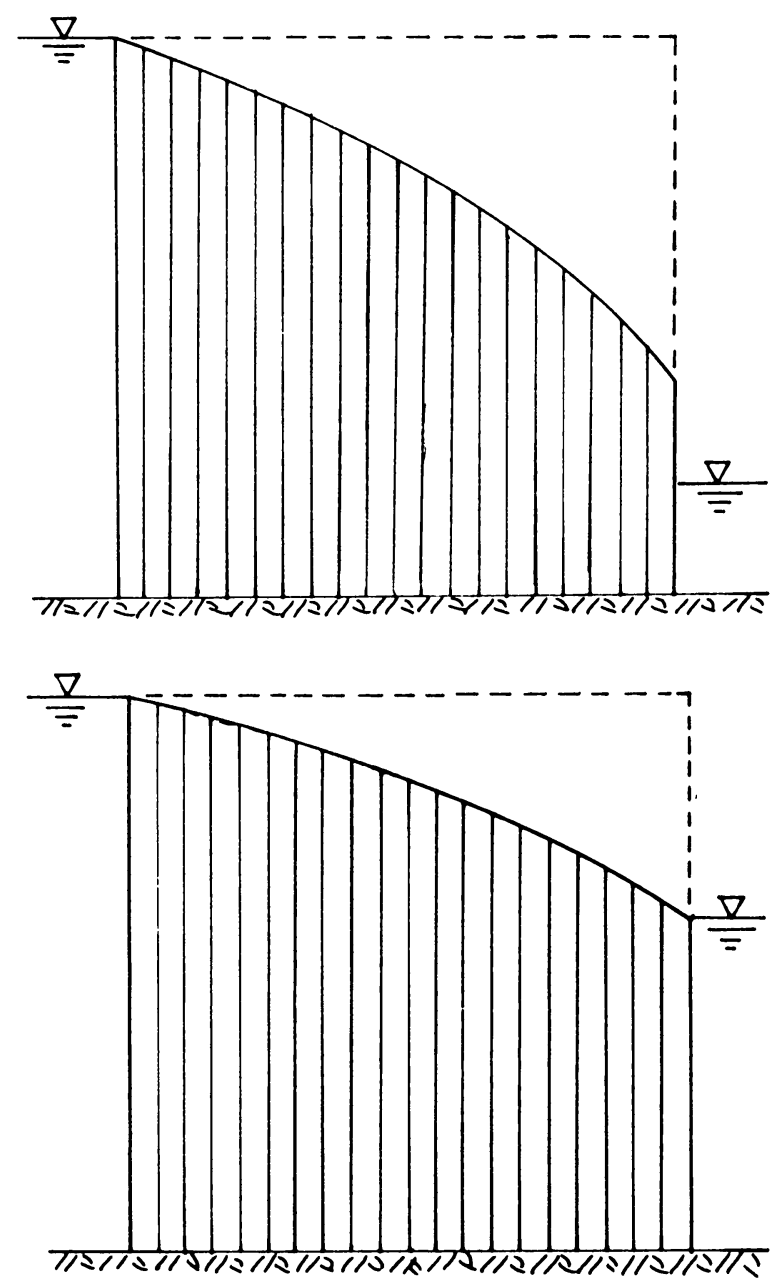

Fig. 6b. Free surface by variational inequality (3200 finite elements).

\section{REFERENCES}

[1] G. Duvaut and J. L. Lions, Sur les inequations en mecanique et physique, Dunod, Paris (1972)

[2] G. Fichera, Problemi elastostatici con vincoli unilaterali: el problema di Signorini con ambigue condizioni al contorno, Atti Acad. Naz, Lincei Mem. Cl. Sci. Fis. Mat. Nat. 8, 91-140 (1964)

[3] J. L. Lions and G. Stampacchia, Variational inequalities, Com. Pure Appl. Math. 20, 493-519 (1967)

[4] J. L. Lions, On free surface problems, in Computational mechanics, Lecture Notes in Mathematics, No. 461, Springer-Verlag, 129-148 (1975)

[5] C. Baiocchi, Su un problema di frontiera libera connesso a questioni di idraulica, Ann. di Mat. Pura ed Appl. (4) 92, 107-127 (1972)

[6] C. Baiocchi, V. Comincioli, E. Magenes, and G. A. Pozzi, Free boundary problems in the theory of fluid flow through porous media, Ann. di Mat. Pure. ed Appl. (4) 97, 1-82 (1973)

[7] C. Baiocchi, Problèmes a frontiere libre en hydraulique, C. R. Acad. Sc. Paris 278, 1201-1204 (1974)

[8] V. Comincioli, Metodi di vilassamento per la minimizzazione in uno spazio prodotto, L.A.N., C.N.R., Pavia. 20, 1971

[9] V. Comincioli, L. Guerri and G. Volpi, Analisi numerica di un problema di frontiera libera connesso col moto di un fluido attraverso un mezzo poroso, Publ. 17 del L.A.N. dell'Universita di Pavia, 1971 
[10] R. S. Falk, Error estimates for the approximation of a class of variational inequalities, Math. Comp. 28, 963-971 (1974)

[11] R. L. Taylor, A. M. Asce and C. B. Brown, Darcy flow solution with a free surface, A.S.C.E., Proc. HY93, 25-33 (1967)

[12] H. Brezis, Problèmes unilateraux, J. Math. Pures et Appl. 51, 1-168 (1972)

[13] C. W. Cryer, The solution of a quadratic programming problem using systematic overrelaxation, SIAM J. Control 9 (1971) 